Herzschr Elektrophys 2018 - 29:244-245

https://doi.org/10.1007/s00399-018-0579-6

Received: 10 June 2018

Accepted: 11 June 2018

(c) Springer Medizin Verlag $\mathrm{GmbH}$, ein Teil von

Springer Nature 2018

CrossMark

\author{
Felix Bourier ${ }^{1,2} \cdot$ Pierre Jais ${ }^{1,2}$ \\ 'Hôpital Cardiologique du Haut-Lévêque, Bordeaux-Pessac, France \\ ${ }^{2}$ IHU Liryc, CHU Bordeaux, Bordeaux-Pessac, France
}

\title{
Mapping of complex arrhythmias: where do we stand and where do we go from here?
}

fluoroscopic imaging alone. Over time, there have been many advances in mapping and imaging technologies, which allowed improvements in the efficacy of ablation procedures. The first generation electroanatomic mapping systems provided basic information about patient anatomy, as well as map color coding for activation and voltage. More recently, a new generation of high-density multielectrode mapping catheters is finding increasing application in clinical prac- tice. As these mapping catheters have the potential to acquire several thousand electrograms within a short time, they are frequently used in combination with computer-assisted mapping algorithms that automatically annotate recorded electrograms. This results in fast, precise, high-density maps with a low level of interpolation, allowing unique insights and visualization of arrhythmia mechanisms (• Fig. 1). However, these recent mapping systems should not be ology procedures were purely guided by

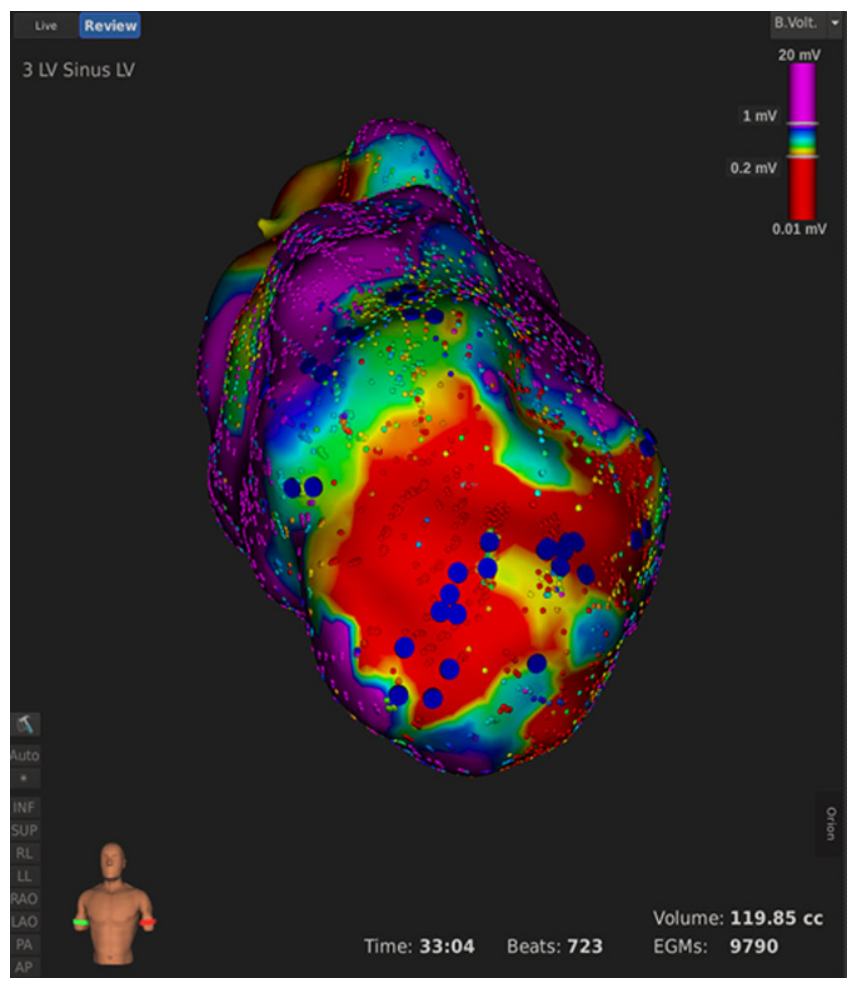

Fig. $1 \Delta$ Anteriorview of high-density left ventricular Rhythmia mapin a patient with ischemic cardiomyopathy delineates an anterior apical scar during a ventricular tachycardia ablation procedure. Blue markers were used to tag identified LAVA (Local Abnormal Ventricular Activity) potentials

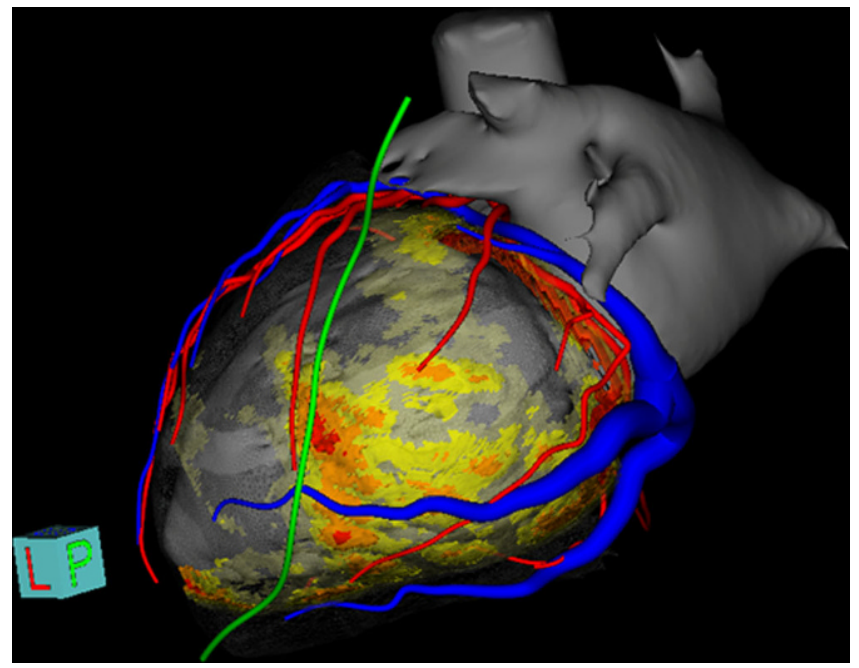

Fig. $2 \Delta$ A myocardial wall thinning (MWT) CT was processed and segmented using the MUSIC software (IHU LIRYC, Bordeaux and Inria, Sophia Antipolis, France, ANR-11-EQPX-0030), pre-procedurally to a ventricular tachycardia (VT) ablation procedure. Left atrium and left ventricle anatomy segmentation is shown in grey. The segmentation of the left phrenic nerve is displayed in green, coronary arteries in red and the coronary venous system in blue. The MWT map (yellow to red color gradient) is used to identify the VT substrate and may intraprocedurally guide the VT ablation procedure 
seen as high density only. A large part of the value comes from the quality of small and shortly coupled electrodes providing a very accurate near field oriented type of mapping. There have been several demonstrations of the superiority of this strategy over mapping with an ablation catheter. Currently, in parallel with recent advances in mapping technologies, the role of cardiac imaging is expanding from a diagnostic tool to one providing specific anatomical guidance for ablation procedures and assessment of arrhythmia substrates (• Fig. 2). As a result, current electroanatomic mapping systems provide real-time image integration techniques, which facilitate the fusion of different imaging modalities. The use of image integration techniques may combine the specific advantages of each modality and confer added value to electrophysiology procedures.

When utilizing advanced mapping technologies in clinical practice, it is important to recognize that their visualization and evidence is still dependent on underlying technologies and data processed by algorithms. A careful review of electrograms and interpretation of acquired maps still remains a mandatory task for electrophysiologists. This should be borne in mind when planning teaching and training programs.

This current issue of Herzschrittmachertherapie und Elektrophysiologie focuses on recent advances and future developments in electrophysiology mapping techniques. Please enjoy the content of these interesting articles!

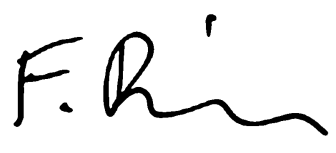

F. Bourier, MD

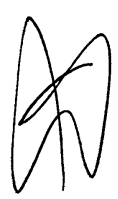

Prof. P. Jais

\section{Corresponding address}

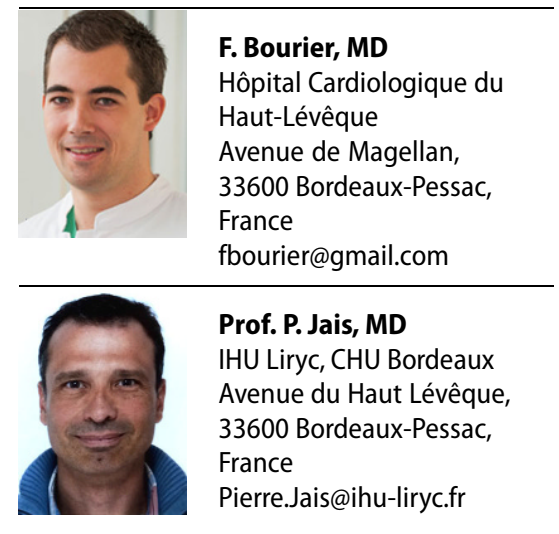

Conflict of interest. P. Jais received consulting fees and speaker honorarium from Boston scientific, Medtronic, Biosense Webster and Abbott. F. Bourier declares that he has no competing interests. 